\title{
Le paradoxe anversois. Entre racisme politique et ouvertures multi-
} culturelles

Hassan Bousetta, Monsieur Marco Martiniello, Madame Marie-Antoinette Hily

\section{Citer ce document / Cite this document :}

Bousetta Hassan, Martiniello Marco, Hily Marie-Antoinette. Le paradoxe anversois. Entre racisme politique et ouvertures multiculturelles. In: Revue européenne des migrations internationales, vol. $14, \mathrm{n}^{\circ} 2,1998$. Immigrés et minorités ethniques dans l'espace politique européen. pp. 151-172;

doi : https://doi.org/10.3406/remi.1998.1635

https://www.persee.fr/doc/remi_0765-0752_1998_num_14_2_1635

Fichier pdf généré le 20/04/2018 


\title{
Résumé
}

Le paradoxe anversois. Entre racisme politique et ouvertures multiculturelles.

Hassan Bousetta.

L'article analyse la participation et la représentation politiques des minorités ethniques aux institutions politiques formelles dans le contexte belge, et en particulier dans la ville d'Anvers. La contribution discute les questions méthodologiques et conceptuelles liées à cette problématique et tente d'esquisser un état des lieux aussi complet que possible de cette représentation politique au plan local. Le phénomène de l'émergence politique des candidats et des élus d'origine ethnique est ensuite étudié. L'hypothèse selon laquelle la forte représentation de l'extrême-droite à Anvers a pu en tant que telle constituer un facteur de réaction politique au sein des groupes ethniques d'origine immigrée est examinée. Une dernière problématique soulevée dans cet article a trait à l'impact d'une telle représentation sur le cours des politiques publiques locales à Anvers.

\begin{abstract}
The Antwerp Paradox. Between Political Racism and Multicultural Perspectives. Hassan Bousetta.

The article examines the political participation and representation of ethnic minorities within Belgian elected assemblies in the city of Antwerp. The contribution endeavours at providing a comprehensive empirical overview of the issue at stake and discusses a number of methodological and conceptual issues related to the study of the political representation of ethnic minorities. The political emergence of ethnic minority candidates and elected politicians is then studied. One aim of the article is to assess whether the presence of a strongly represented extreme-right, as it is the case in Antwerp, has constituted a factor of political reaction within immigrant ethnic communities in Antwerp. Another issue raised in this contribution is about the impact of the political representation of ethnic minorities on the course of local public policies.
\end{abstract}

\section{Resumen}

La parádoja de Amberes. Entre racismo político y aperturas multiculturales.

Hassan Bousetta.

Esta contribución tiene por objeto el análisis de la participación y de la representacion políticas de las minorias étnicas en las instituciones políticas formales en el contexto belga. Aunque esta representación de las minorias étnicas es todavía un fenomeno cuantitativamente limitado en el seno de multiples asambleas elegidas por el Estado belga, sin embargo, se trata de un fenómeno político emergente en el contexto de los años noventa. El estudio de esta problemática se concentra en el caso particular de la ciudad de Amberes. La contribución, que presenta un carácter introductorio, discute las cuestiones metodológicas y conceptuales unidas a esta problemática e intenta esbozar un estado de la cuestion tan completo como sea posible de esta representación politica en el plano local. A continuación, se estudia el fenómeno de la emergencia política de los candidatos y de los elegidos de origen étnico, desde el punto de vista de sus diferentes modalidades en el contexto de Amberes. En efecto, una de las hipótesis que nos ha guiado, en relación con este trabajo, ha consistido en estudiar en qué medida la fuerte representación de la extrema-derecha en Amberes ha podido, como tal, constituir un factor de reaccion política en el seno de los grupos étnicos de origen inmigrado. Una ultima problematica planteada en este artículo se refiere al impacto de una representación, como ésta, en el desarrollo de las políticas públicas en Amberes.

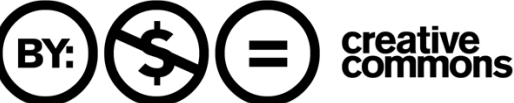




\title{
Le paradoxe anversois Entre racisme politique et ouvertures multiculturelles
}

\author{
Hassan BOUSETTA*
}

Cet article ${ }^{1}$ a pour objet l'analyse de la participation et de la représentation politique des minorités ethniques aux institutions politiques formelles dans le contexte belge $^{2}$. Bien que la représentation de cette population reste un phénomène quantitativement limité au sein des multiples assemblées élues de l'Etat belge, il s'agit néanmoins d'un phénomène politique émergeant dans le contexte des années 90 . Nous avons choisi ici d'explorer les caractéristiques de cette question à partir d'une étude de cas, celui de la ville d'Anvers.

Au sein de l'espace belge et flamand, Anvers présente un profil particulier au plan des réalités politiques. En effet, la ville a connu au tournant des années 90 le début d'une période de mutations profondes et durables de son paysage politique. Ces

* Chercheur à la Katholieke Universiteit Brussel, Faculteit Politieke en Sociale Wetenschappen, Centrum voor Toegepast Multivariaat Onderzoek, 17, Vrijheidslaan 1081 Brussel, België. Email: Hassan.Bousetta@kubrussel.ac.be

1 Je tiens à remercier Marc Swyngedouw, Dirk Jacobs ainsi que les éditeurs de ce numéro pour leurs remarques et commentaires sur une version antérieure de cette contribution.

2 Nous préférerons ici l'utilisation du terme « minorités ethniques » à d'autres appellations pour caractériser les populations issues de l'immigration. Malgré ses imperfections, ce concept a l'avantage de faire la distinction entre les groupes de migrants structurellement dominés dans la société flamande et anversoise et les populations migrantes présentant des profils socioéconomiques plus avantagés. Elle nous permet également de ne pas nous enfermer dans les classifications dichotomiques tributaires du critère de la nationalité (i.e. national-étranger). Dans le cadre du travail de terrain que nous avons mené ici, nous nous sommes toutefois prioritairement intéressés aux groupes problématisés dans le débat public, à savoir essentiellement les Marocains et les Turcs. Deux autres groupes qui à eux seuls méritent une analyse approfondie sont exclus du champ d'observation : la communauté juive anversoise et le groupe de population néerlandais (ou d'origine néerlandaise), qui historiquement a toujours été fortement représenté à Anvers et dans la périphérie anversoise. 
mutations, toujours en cours, sont liées aux percées électorales successives d'une formation politique d'extrême-droite, le Vlaams Blok, dont le programme politique repose en grande partie sur la manipulation de slogans racistes anti-immigrés. Le dividende électoral que le Vlaams Blok est parvenu à tirer de l'utilisation de cette rhétorique raciste l'amène aujourd'hui à constituer, avec $28 \%$ des suffrages lors des dernières élections communales de 1994 , la première force politique anversoise. A partir de ses succès électoraux à l'occasion des élections communales de 1988 et de 1994, et des élections législatives de 1991 et de 1995, cette formation a essaimé bien au-delà de sa base historique anversoise et a consolidé ses positions dans l'ensemble de la région flamande.

L'émergence de l'idéologie de fermeture prônée par le Vlaams Blok et sa capitalisation électorale contrastent fortement avec les réalités objectives de la dynamique urbaine anversoise. Au plan humain, culturel, et économique, l'ouverture de la société anversoise l'emporte largement. Accueillant le plus grand centre portuaire du pays, et le deuxième en importance au niveau européen, Anvers se trouve au coeur d'échanges économiques mondiaux, qui, aujourd'hui comme hier, contribuent grandement à sa prospérité. Les divers courants de populations migrantes qui se sont installées à Anvers tout au long du siècle ont largement modifié la composition démographique de la ville. Avec près de $13 \%$ de population non-belges issues d'une multitude de communautés immigrées, Anvers est une ville extrêmement diversifiée au plan culturel ${ }^{3}$ et humain.

La confrontation entre l'idéologie de la «société fermée » incarnée par le Vlaams Blok et l'ouverture bien réelle du microcosme anversois forme la trame de fond de ce qu'on pourrait qualifier de paradoxe anversois. Au plan politique, le dévoilement de ces orientations contradictoires a fait l'objet de nombreuses analyses minutieuses, qui ont permis de mettre en évidence un élément explicatif crucial dans l'évolution de la dynamique politique locale (Swyngedouw, 1998). La transformation du paysage politico-électoral se produit dans le contexte d'une mutation plus profonde des modes de régulation de la société. Le reflux électoral de la toute puissance des formations politiques de centre-droit (chrétiens-démocrates) et de centre-gauche (socialistes) consacre la fin de l'équilibre particratique et clientéliste qui était au fondement de leur hégémonie. Ce déclin des formations du centre s'accompagne en l'occurrence d'une progression spectaculaire de la représentation des forces de l'extrême-droite d'une part, mais également par une progression significative d'un parti écologiste tenant d'une vision multiculturelle d'autre part.

L'émergence proprement politique de la question de l'immigration est donc davantage un révélateur que la cause objective des mutations en cours dans la société anversoise et dans la société belge toute entière. Le contexte anversois est, de ce point de vue, un objet d'étude pertinent parce que les contours de cette transformation y sont particulièrement visibles. Considéré, pour l'observateur, comme un laboratoire, il

3 Au plan artistique et culturel, la vitalité de la ville, qui fut en 1993 capitale culturelle européenne, n'est pas à démontrer. 
permet de mettre en évidence quelques uns des enjeux politiques fondamentaux liés, plus généralement, à la gouvernance des grands centres urbains multiculturels. Nous examinerons dans cet article l'une des dimensions de ces réalités politiques émergentes : la stigmatisation des minorités ethniques comme stratégie de mobilisation politique et l'affirmation d'une réaction politique par les minorités elles-mêmes au sein des institutions politiques formelles. Il s'agit de voir dans quelle mesure l'émergence d'une extrême-droite politiquement organisée et représentée dans les enceintes élues a pu représenter dans le contexte anversois un facteur de mobilisation et de réaction politique au sein des groupes ethniques immigrés ${ }^{4}$.

\section{LE CONTEXTE POLITIQUE ANVERSOIS}

L'espace politique anversois a été dominé à partir du début des années 20 par l'influence conjuguée des courants socialistes et sociaux-chrétiens. Comme le rappelle M. Swyngedouw (1998), la coalition formée par ces deux partis politiques a, par delà les aléas de l'histoire de ce siècle, réussi à maintenir une présence ininterrompue au pouvoir dans la ville pendant plus de soixante-dix ans. Pour comprendre la logique de la longévité politique de l'alliance entre socialistes et sociaux-chrétiens, il est nécessaire d'éclairer le contexte social plus large dans lequel ce phénomène s'inscrit. La stabilité de cette hégémonie politique s'est en réalité longtemps appuyée sur une multitude de relais dans la société civile, laquelle se caractérisait par une organisation « en piliers » (Van den Brande, 1967 ; Martiniello et Swyngedouw, 1998). Ce système propre à l'ensemble du pays s'articule autour d'une série de trois clivages, dont un clivage philosophico-religieux démarquant un univers social laïc de tendance socialiste et un univers social chrétien ${ }^{5}$. La gestion de la cohabitation entre les populations appartenant à ces univers cloisonnés s'effectuait en retour à travers la méthode du compromis au niveau des élites politiques. La logique de fonctionnement du système, essentiellement clientéliste, a pu garantir un contrôle politique effectif des bases, lequel s'est traduit par une relative immunité vis à vis des possibilités de sanction électorale.

L'érosion de ces modes de gestion des conflits politiques et sociaux, à la fois particratique et clientéliste, semble inéluctable dès le milieu des années $80^{6}$.

4 L'action politique des candidats et des élus d'origine ethnique est interprétée par certains comme un fait politique relevant de l'initiative individuelle, et, à ce titre, dissociable des dynamiques collectives à l'oeuvre au sein des collectivités ethniques. Nous continuons à penser avec C. Withol de Wenden (1995) que ces phénomènes sont à un certain niveau indicatifs des stratégies collectives des minorités. On pourrait même avancer que dans un certain nombre de cas, l'action politique individuelle et l'action politique collective sont des variantes l'une de l'autre.

5 Les deux autres clivages sur lesquels s'est forgée la société belge actuelle sont le clivage linguistique et communautaire et le clivage possédants-travailleurs.

6 Parmi les éléments qui annoncent cette crise au niveau proprement anversois, Marc Swyngedouw relève la crise des finances publiques locales, la modification de la composition démographique de la population de la ville résultant d'une part d'un phénomène d'exode vers la périphérie et d'autre part d'une immigration en provenance soit d'autres régions flamandes soit d'autres pays. 
Cependant, la traduction des mutations en cours dans la société locale en terme de changement sur la scène politique et électorale ne vont émerger de manière brutale qu'à la fin des années 80 et au début des années 90 . Les partis politiques traditionnels dominant la scène anversoise, socialistes et sociaux-chrétiens, subissent un premier choc d'envergure à l'occasion des élections communales d'octobre 1988. L'électorat des socialistes et des sociaux-chrétiens s'est pour la première fois significativement déplacé au profit de l'extrême-droite, laquelle quintuple son résultat en siège au niveau du conseil communal. Cette première défaite des partis démocratiques en annonce d'autres. Aux élections législatives de 1991, alors que le gouvernement national est engagé dans la négociation d'un nouveau train de réformes constitutionnelles qui débouchera sur l'avènement d'un Etat fédéral, le discours raciste du Vlaams Blok fait à nouveau mouche? ${ }^{7}$.

Entre 1988 et 1991 , la réaction des partis politiques traditionnels, et principalement du CVP (chrétiens-démocrates), du SP (socialistes), et de la VU (nationalistes démocratiques), est incertaine. Leur tactique consistera tout d'abord à reprendre en main la question de l'immigration en imposant au niveau national, en mars 1989, la création d'un commissariat royal à la politique des immigrés. Cette institution consultative, à la tête de laquelle sera désignée une ex-Ministre du parti social-chrétien flamand, assistée par un commissaire royal adjoint socialiste bruxellois, se verra confier par le gouvernement national la mission d'examiner et de proposer les mesures qui s'imposent à l'égard de la problématique des immigrés. En déplaçant ainsi le traitement de cet enjeu vers les sphères décisionnelles nationales, les partis menacés sur le terrain par la montée de l'extrême-droite cherchent avant tout à définir une ligne d'action politique consensuelle afin de neutraliser et de désamorcer le thème de l'immigration en tant qu'enjeu électoral.

Dans le même temps cependant, des voix se font entendre au sein de ces mêmes partis, et plus singulièrement au niveau local, pour imposer une rhétorique de fermeté vis-à-vis des populations immigrées installées et vis-à-vis des nouveaux arrivants. Alors même que les commissaires royaux défrichent l'ensemble des problématiques liées à l'intégration des immigrés en formulant un ensemble impressionnant de 262 propositions, on assiste simultanément à une forme d'irradiation des thèses d'extrême-droite au niveau local. Les populations immigrées et principalement les populations musulmanes ressortissantes des pays non-européens, Marocaines et Turques, sont de plus en plus prises pour cible. Leur stigmatisation trouvera des illustrations particulièrement spectaculaires, et ce non seulement dans le discours de l'extrême-droite, mais également dans les discours de quelques élus des partis traditionnels ${ }^{8}$.

7 Les succès électoraux du Vlaams Blok sont largement déterminés par son positionnement en matière d'immigration. Néanmoins, il s'agit également d'un parti qui développe très clairement un programme anti-Belge et séparatiste en faveur d'une Flandre indépendante.

8 On peut à cet effet mentionner les dérives racistes de la campagne électorale du candidat Fernand Huts, candidat libéral de droite à Anvers lors des élections législatives de 1995. Bien

REMI 1998 (14) 2 pp. 151-172 
Le succès le plus imposant de l'extrême-droite au tournant des années 90 aura été de parvenir à imposer l'immigration comme un enjeu essentiel de la politique anversoise et, par voie de conséquence à influencer les stratégies partisanes de l'ensemble des partis démocratiques. Les tentatives d'isoler la question, par la voie technico-administrative via le Commissariat Royal s'avéreront du point de vue strictement électoral un échec. En effet, deux ans après la création de cette institution consultative, à l'occasion des élections législatives de 1991, le Vlaams Blok sera pour les partis démocratiques traditionnels l'origine d'un deuxième « dimanche noir ».

Lors des élections communales de 1994, la rupture entre les formations socialistes et sociales-chrétiennes d'une part et leur base électorale traditionnelle d'autre part est définitivement consommée. L'extrême-droite supplante l'ensemble des partis politiques démocratiques et s'impose comme première force politique anversoise avec $28 \%$ des suffrages. Alors que les socialistes chutent à leur niveau le plus bas d'après-guerre, les sociaux-chrétiens qui s'étaient présentés devant les électeurs en ordre dispersés maintiennent une présence très affaiblie au sein d'une liste de cartel (Antwerpen 94) aux côtés des nationalistes démocratiques de la VU. Les écologistes d'Agalev de leur côté maintiennent une progression constante par rapport aux deux précédents scrutins communaux.

Ces indications traduisent la profonde mutation des attitudes de l'électorat anversois et flamand plus généralement (Swyngedouw, 1992). Avec un système électoral essentiellement basé sur la représentation proportionnelle, on comprendra que le Vlaams Blok n'est aujourd'hui maintenu aux marges du pouvoir local que par la seule volonté de l'ensemble des formations politiques démocratiques de s'unir dans une «coalition des forces démocratiques ». La formation de l'exécutif communal issue de ce scrutin n'aura en effet d'autre choix que celui d'imposer une stratégie du « cordon sanitaire » visant à faire barrage à l'extrême-droite. Dès lors, socialistes, chrétiens-démocrates, nationalistes démocratiques, libéraux de droite et écologistes vont se trouver contraints de gérer la ville au sein d'une coalition politique hétéroclite. Malgré la diversité des courants politiques représentés dans cette coalition anti-Vlaams Blok, l'un des éléments essentiels de la nouvelle politique communale anversoise qui se met en place en 1994 tient dans sa relative ouverture aux questions d'immigration et de multiculturalisme.

Alors que le Vlaams Blok maintient sa pression sur les partis démocratiques par une forte politisation du thème de l'immigration, systématiquement amalgamé aux thèmes de l'ordre et de la sécurité, quelques personnalités issues des minorités ethniques émergent sur la scène politique et électorale. Il s'agit là d'une des

que le parti libéral de droite se soit le plus fortement laissé influencé par le discours de l'extrême-droite, le parti socialiste et le parti social-chrétien ne seront toutefois pas à l'abri de ce même type de dérives. 


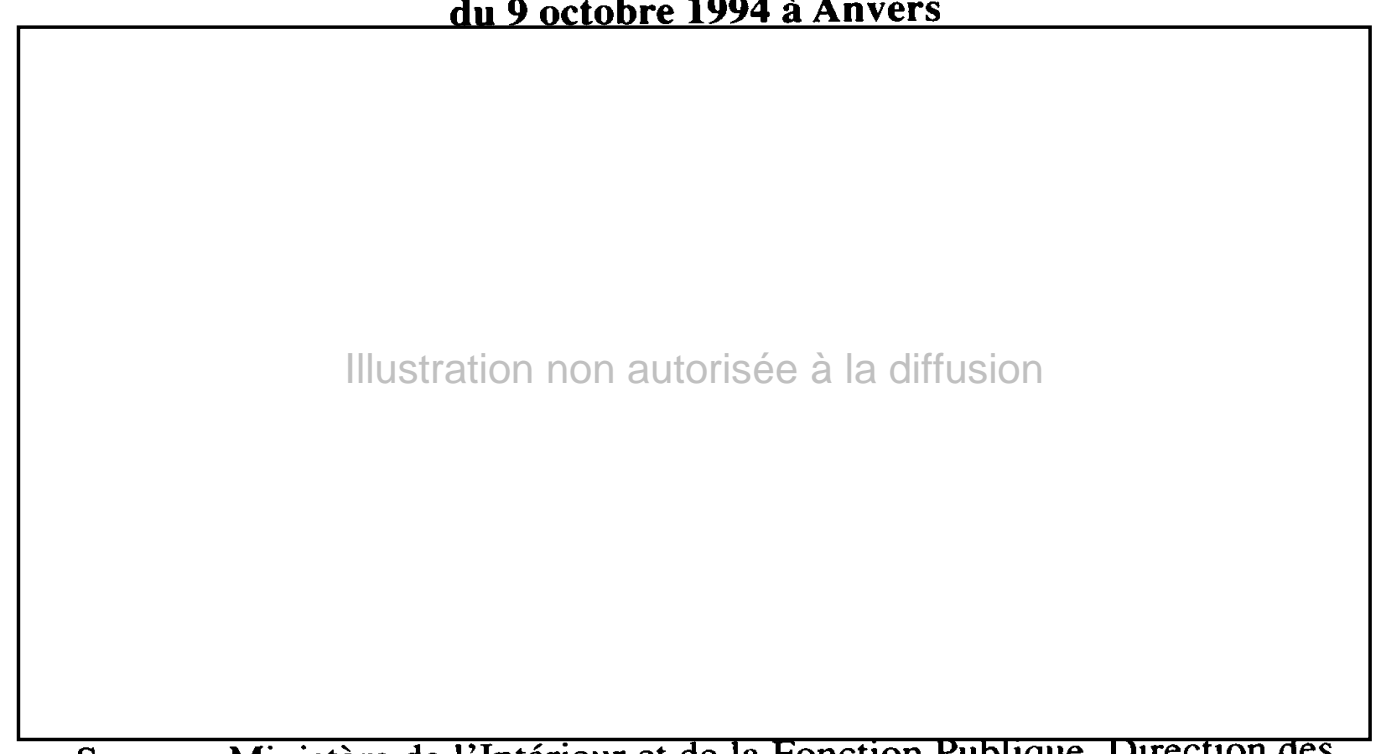

Source : Ministère de l'Intérieur et de la Fonction Publique, Direction des élections et de la population.

observations significatives par rapport aux derniers scrutins, dont le scrutin communal d'octobre 1994 (Blaise et Lentzen, 1994). L'apparition de candidats d'origine étrangère sur les listes électorales commence à s'imposer dans l'ensemble du pays, confirmant les premiers signes déjà perceptibles en 1988 : Fatima Bali, candidate écologiste d'origine marocaine fut élue en 1988 à Anvers. Elle sera réélue en 1994 et rejointe dans la nouvelle assemblée par une autre candidate d'origine marocaine, Nahima Lanjri, élue parmi les candidats sociaux-chrétiens de la liste Antwerpen 94 . Au-delà de la forte charge symbolique associée à la réussite individuelle de ces deux femmes, appartenant à la jeune génération de l'immigration d'origine marocaine, leur engagement politique et leur élection signalent un changement dans les stratégies de réaction politique des minorités ethniques. L'investissement de l'arène électorale, qui avait été inexistant pour les personnes de la première génération, faute de disposer de droits politiques équivalents à ceux des Belges, devient une option de plus en plus sérieuse.

\section{PARTICIPATION ET REPRÉSENTATION POLITIQUES DES MINORITÉS ETHNIQUES : LES FACTEURS DE CONTINUITÉ ET DE CHANGEMENT}

\section{Participation et représentation politiques des minorités ethniques : un point de méthode 9}

En Belgique, l'évaluation quantitative du phénomène de la participation et de la représentation politiques des minorités ethniques pose de sérieuses difficultés de

9 Nous adoptons ici une définition délibérément étroite de la participation politique. Il s'agit principalement de ce que Marco Martiniello qualifie de participation politique étatique, à savoir la participation électorale et la participation par la représentation dans les assemblées élues (M. Martiniello, 1997 : 111-117). 
méthode. Du point de vue de la participation politique, aucune source d'information ne nous permet d'établir un quelconque diagnostic. A ce stade, il nous faut préciser que la population que nous qualifions de minorités ethniques est essentiellement composée de personnes issues de l'immigration, qui sont majoritairement de nationalités étrangères. Ces personnes sont donc exclues du bénéfice du droit de vote et d'éligibilité. Dès lors, l'étude de leur participation politique électorale trouve une restriction de taille dans le contexte belge puisqu'elle ne peut s'intéresser qu'aux phénomènes touchant les personnes ayant acquis la nationalité belge. Au niveau de l'ensemble du pays, on estime que la population qui est passée par les diverses procédures de naturalisation tout à la fois par les procédures d'attribution et par celles d'acquisition de la nationalité belge - depuis 1985 s'élève à plus de 250.000 personnes ${ }^{10}$. Cependant, malgré l'importance quantitative sans cesse croissante de cette population, la nature de leur comportement électoral effectif reste à ce jour une énigme. Contrairement aux techniques de recherche, du type sondage sortie des urnes ou survey, utilisées dans les grands pays multiculturels pour évaluer le comportement électoral des minorités ethniques et raciales, aucune étude en Belgique ne nous renseigne à ce jour sur ce sujet $^{11}$.

Du point de vue de la représentation, par contre, un certain nombre d'informations et de données peuvent être établies. Une telle analyse se doit néanmoins de poser un certain nombre de jalons méthodologiques. En effet, l'évaluation quantitative de l'émergence des candidats et des élus issus de cette catégorie de la population ne peut être utilement développée qu'à partir de différentes méthodologies, dont seule la combinaison permet d'exploiter les sources disponibles. Etant donné que ces candidats ne sont en aucune manière distingués officiellement, les observateurs doivent pour procéder à leur identification combiner une analyse patronymique à une analyse biographique par interview directe. En complément, d'autres méthodes plus classiques s'imposent, telles l'analyse de contenu des archives électorales, l'analyse de contenu de la presse et des publications électorales des partis politiques.

Parmi ces méthodes, l'approche patronymique initiale est certainement la plus problématique. Elle vise en principe à établir un repérage des noms à consonance étrangère inscrits sur les listes électorales. Cette méthode est pour de multiples raisons hautement incertaine, voire contestable. L'identification des origines ethniques des

10 Ces 250.000 unités rassemblent donc l'ensemble des personnes qui sont passées de la catégorie statistique «Belge » à la catégorie « étrangers ». Par ailleurs la Belgique comptait, au 01-01-1996, une population étrangère totale s'élevant à 909.769 personnes, dont 554.517 Européens et 355.252 Non-Européens. Voir Centre pour l'Egalité des Chances, « Rapport Annuel. Annexe Informative », Bruxelles, 1996.

11 La sensibilité politique des questions dites communautaires, à savoir la question des divisions ethnico-linguistiques, a poussé certains chercheurs à évaluer le comportement électoral éventuel de la population européenne résidant dans la périphérie flamande de Bruxelles. Cette étude ne s'intéresse toutefois prioritairement qu'à la question de l'offre politique des partis du point de vue linguistique. En d'autres termes, cette étude cherchait à déterminer la propension de l'électeur de nationalité européenne à voter pour un parti francophone ou pour un parti flamand. 
candidats ne peut en effet produire aucun résultat fiable si elle se base exclusivement sur la patronymie. L'analyse patronymique pose dans la pratique toute une série de problèmes parmi lesquels la question centrale du changement de nom par le mariage, qui, en Flandre, trouve une complexité supplémentaire due à une latinisation très répandue des prénoms féminins.

Par ailleurs, l'entreprise visant à identifier les origines ethniques des candidats et des élus politiques peut se heurter à une opposition plus fondamentale sur son statut en tant qu'instrument de recherche et sur ses visées théoriques. Cette opposition est particulièrement évidente si l'on se place dans la perspective normative assimilationiste, qui considère les phénomènes identitaires liés à l'ethnicité comme des phénomènes transitoires et temporaires. Une autre critique consiste à avancer que de telles études mènent inévitablement à développer des visions primordialistes et chosifiantes de l'ethnicité des candidats et des élus politiques : une vision qui tendrait en quelque sorte à faire des candidats et des élus politiques issus des minorités ethniques, des candidats et des élus ethniques (voir Taylor, 1996).

Quand bien même, on s'affranchit de la critique assimilationiste en lui substituant un autre présupposé normatif reposant sur l'idée que les grands centres urbains européens sont confrontés à des logiques allant dans le sens d'une ethnicisation du champ politique, on ne peut complètement ignorer le deuxième volet de la critique de ces méthodes visant à établir les origines ethniques des politiciens. L'ethnicité et son affirmation dans l'espace politique n'étant pas les produits d'un "état de nature ", on ne peut uniquement considérer l'étendue de la représentation numérique de ces populations, sans poser que la classification des origines ethniques est en soi un exercice problématique (Stasiulis, 1997).

Dans l'état actuel des choses, l'explicitation des présupposés normatifs, la multiplication des stratégies de recherche, des méthodes d'analyse des données, et des sources d'information peut constituer une réponse adaptée aux obstacles théoriques et méthodologiques. Mais ce type d'approche est en réalité plus qu'une exigence positive. En effet, la non prise en compte de cette exigence est susceptible d'orienter l'ensemble de la perspective théorique et normative. Ce sont ces aspects implicites au processus de construction de l'objet que nous avons voulu souligner afin de montrer qu'une analyse de la représentation politique de ces groupes ne prend son sens qu'en tant qu'élément d'une étude plus large sur leurs stratégies de mobilisation et d'influence politique. En d'autres termes, l'analyse de la représentation numérique des minorités ethniques ne peut être déconnectée d'une analyse de la représentation de leurs intérêts politiques. C'est pour cette raison que, dans le cadre de cette contribution particulière, nous avons délibérément restreint le champ de notre analyse aux situations permettant d'éclairer l'apparition d'identités et de revendications politiques différenciées dans l'espace local. Ce qui nous a guidé par rapport au terrain anversois consistait à voir dans quelle mesure s'opèrent des stratégies de mobilisation de l'ethnicité et dans quelle mesure ces stratégies sont elles-mêmes tributaires de la radicalisation des débats imposés par les succès électoraux massifs de l'extrême-droite. 
En fonction de ces repères, nous avons, dans notre étude du cas anversois, maintenu l'analyse patronymique en tant qu'indicateur primaire. Nous l'avons complétée par une analyse biographique, grâce à une série d'entretiens réalisés avec un groupe de témoins privilégiés composé de candidats et d'élus issus des minorités ethniques. En outre nous avons également mobilisé les approches qualitatives classiques de l'étude du politique. Nous avons ainsi mené une série d'entretiens avec les responsables des partis politiques locaux et nationaux, une analyse de contenu qualitative des archives électorales, et une analyse documentaire des programmes des partis politiques.

\section{Participation et représentation politique des minorités ethniques : esquisse d'un état des lieux}

Bien que nous nous soyons prioritairement attaché à l'étude de la situation politique communale anversoise, il nous a semblé utile d'étendre notre analyse aux résultats enregistrés dans la région anversoise à l'occasion d'élections autres que locales, ceci afin de faire apparaître les grandes tendances. Au cours de la période 1988-1998, la Belgique a en effet connu un cycle électoral comprenant près d'une dizaine de scrutins. Ceux-ci visaient le renouvellement des conseils communaux (1988-1994), des conseils provinciaux (1991-1994), de la Chambre (1991-1995), du Sénat (1991-1995), la désignation des représentants au Parlement Européen (19891995) et la première élection directe des conseils régionaux institués par la Constitution fédérale (1995). Toutes précautions méthodologiques gardées, trois constatations s'imposent quant à l'analyse strictement électorale de la participation et de la représentation politiques des groupes issus de l'immigration au cours du cycle électoral 1988-1995.

Un premier constat concerne l'échelon communal. A l'occasion des élections d'octobre 1994, la représentation des personnes d'origine étrangère, non ressortissantes d'un pays de l'Union Européenne, au sein des conseils communaux au niveau de l'ensemble de la Flandre est passée de 2 à 13 élus. Ces résultats ne traduisent cependant qu'une partie du phénomène observé lors des derniers scrutins. En effet, malgré le fait que seuls, 13 élus aient franchi le cap de la représentation, ils étaient en amont beaucoup plus nombreux à briguer les suffrages de l'électeur. Au niveau de la Flandre, on peut estimer que lors du scrutin communal d'octobre 1994 le nombre de candidats non-élus issus de ces groupes de population s'élevait à plus d'une centaine ${ }^{12}$.

12 Il est techniquement très difficile d'obtenir sur ce point des données autres que des estimations relativement imprécises. Il faudrait, pour parvenir à une estimation fiable, consulter l'ensemble des listes électorales présentées dans les 303 communes de Flandre. Or ces listes électorales officielles, qui sont archivées selon la procédure légale au greffe des administrations provinciales ainsi qu'aux tribunaux de première instance, sont dans la pratique d'un accès malaisé. 
A Anvers, nous avons pu identifier, sur la base des listes électorales présentées lors des élections communales de 1994, 12 candidats d'origine non-européenne, tous d'origine Marocaine ou Turque à une exception près. Parmi ceux-ci, et comme nous l'avons déjà souligné, seules 2 candidates seront élues au sein du nouveau conseil communal anversois. En ce qui concerne les élections aux autres niveaux de pouvoir dans la région anversoise, elles ne donneront lieu à l'élection d'aucun candidat issu des minorités ethniques, malgré les nombreuses tentatives enregistrées. Cette observation montre la sous-représentation numérique de ces populations malgré les bons résultats obtenus par ces candidats (voir infra).

La deuxième observation met en évidence l'orientation préférentielle de l'électeur anversois vis-à-vis des candidats d'origine ethnique. Rappelons qu'en Belgique, l'électeur peut émettre son suffrage de plusieurs manières. S'il juge satisfaisant l'ordre de présentation des candidats proposés par le parti qu'il choisit, il peut émettre un vote pour l'ensemble de la liste à travers le vote dit de la « case de tête ». Dans le cas où l'ordre de présentation des candidats ne lui convient pas, il peut également exprimer un vote préférentiel soit pour un candidat, soit pour plusieurs candidats d'une même liste, peu importe que ceux-ci soient effectifs ou suppléants. Ce mécanisme du vote préférentiel permet, bien que dans une faible mesure ${ }^{13}$, de modifier l'ordre de présentation des candidats et donc de peser sur leur chance d'être élu. Dans le cas des candidats que nous avons recensés, l'analyse du vote préférentiel s'avère assez intéressante. Ceux-ci réussissent en effet des performances généralement supérieures à la moyenne.

Plusieurs exemples permettent d'illustrer ce point. Lors des élections provinciales de 1991, le parti écologiste Agalev présentait, dans l'arrondissement d'Anvers, un candidat d'origine marocaine, Hocine Souidi, en septième position. Celui-ci réalisera le troisième résultat de son parti au nombre des voix de préférence. Le même scénario se produisit pour Touria Chakri, une candidate d'origine marocaine du parti écologiste lors des élections provinciales à Anvers en 1994. Placée en huitième position, elle obtint le troisième résultat en voix de préférence. Ni l'un ni l'autre ne seront finalement élus. Néanmoins, le trait saillant de ces résultats réside dans le fait que ces deux candidats étaient au moment de leur candidature inconnus dans l'espace politique local. Par ailleurs, ces deux candidats se présentaient au nom d'une formation écologiste où la personnalisation des campagnes électorales est fortement évitée et où le vote préférentiel est traditionnellement faible.

Trois personnalités anversoises se démarquent toutefois de ce schéma et contrastent au contraire par leur forte médiatisation. Il s'agit des deux élues d'origine marocaine au sein du conseil communal, l'écologiste Fatima Bali et la chrétiennedémocrate Nahima Lanjri, et de Mohamed Sebbahi ${ }^{14}$, candidat écologiste aux élections

13 Les modifications autorisées par ce mécanisme sont toutefois plus importantes au niveau des élections communales qu'aux élections aux autres niveaux de pouvoir.

14 Mohamed Sebbahi est l'une des figures politiques marquantes de la communauté marocaine à Anvers et en Flandre de manière plus générale. Mohamed Sebbahi offre un exemple 
législatives de 1991 et de 1995 et aux européennes de 1995. Lors des élections communales de 1994, Nahima Lanjri, placée en huitième position réalise le sixième résultat de sa liste en voix de préférence. Elle réalisera à nouveau un excellent résultat lors des élections régionales de $1995^{15}$. Le cas de Fatima Bali, conseillère communale écologiste de 1988 à 1994 et briguant un nouveau mandat lors des élections de 1994, est particulièrement intéressant puisque son résultat personnel lui a permis de bénéficier du vote préférentiel ${ }^{16}$.

Les succès personnels réalisés par ces candidats sont toutefois indissociables de l'importance des partis auxquels ils appartiennent. En effet, on ne peut observer de tels résultats que lorsque ces candidats appartiennent aux grandes formations démocratiques, à savoir principalement les écologistes, les chrétiens-démocrates, et les socialistes. Il faut en effet souligner que ces candidats ne reproduisent pas ces succès personnels lorsqu'ils se présentent sur des listes marginales, dissidentes ou contestataires. L'exemple des candidats du parti d'extrême-gauche PVDA est à cet égard révélateur. Présents à toutes les élections depuis près de dix ans, et révélant comparativement une certaine continuité dans leur engagement politique, un candidat d'origine turc et une candidate d'origine marocaine n'ont jamais dépassé les quelques centaines de voix de préférence.

D'un point de vue méthodologique, et en l'absence de données résultant d'un sondage (à la sortie des urnes), il est impossible de rendre compte du profil de l'électeur qui porte son choix vers ces candidats. Plusieurs hypothèses d'interprétation méritent néanmoins d'être envisagées. Tout d'abord, on ne pourrait exclure l'idée que le vote pour des candidats d'origine étrangère est d'abord le fait de la population majoritaire anversoise. Dans le contexte d'une forte représentation de l'extrême-droite, le vote pour un tel candidat peut en soi constituer une motivation politique subjective supplémentaire pour les segments de l'électorat des partis traditionnels les plus attachés à l'idéologie multiculturaliste. Dans le contexte de crise de la représentation que traversent la plupart des institutions électives, une volonté de renouveau incarnée par la sélection d'un personnel politique vierge de toute expérience politique antérieure peut également constituer un facteur d'attrait auprès de ce même électorat belge.

particulièrement intéressant d'un ex-candidat aux élections d'origine ethnique et ex-cadre de parti politique, reconverti dans le monde associatif. Après avoir milité pour le principe de la reconnaissance officielle de l'auto-organisation des minorités ethniques, il est devenu directeur de la Federatie Marokkaanse Democratische Organisaties, qui est précisément l'une des neufs fédérations d'associations ethno-culturelles reconnue et financée par le gouvernement flamand depuis 1996.

15 Elle réalise à cette occasion à nouveau le sixième résultat de sa liste en voix de préférence, alors qu'elle était placée en dix-neuvième position et derrière les ténors nationaux du parti, emmenés par le président fédéral des sociaux-chrétiens flamands Marc Van Peel.

16 Placée en neuvième position sur la liste de son parti, son positionnement ne lui permettait pas a priori d'espérer figurer parmi les élus de son parti. Alors que les écologistes ne comptent que sept sièges au sein de l'actuel conseil communal, elle sera toutefois élue grâce au renversement de l'ordre des candidats permis par le vote préférentiel. 
Une autre hypothèse consisterait à expliquer les succès électoraux de ces candidats par l'émergence d'un vote ethnique, dans le sens où le vote individuel de l'électeur issu des minorités ethniques serait décisivement motivé par l'offre ethnique représentée par les candidats, et ce peu importe que ces derniers revendiquent ou non cette représentativité. Les observations relevées permettent d'émettre un doute quant à cette hypothèse. Comme nous l'avons suggéré plus haut, la taille et l'idéologie des partis, la notoriété et l'ordre de présentation des candidats sont des facteurs qui tendent à l'emporter sur une simple logique ethnique. Cependant, on ne peut pas, dans l'état actuel des choses, exclure complètement la variable « ethnicité » de l'analyse électorale. Il faudra en réalité à l'avenir considérer les effets d'une double variable sur le comportement électoral de la population : la variable « ethnicité »d'une part et la variable « ethnicité des candidats »d'autre part. On pourrait en effet maintenir que l'électeur issu des minorités ethniques serait, dans une mesure encore à déterminer, enclin à apporter son soutien, à travers un vote préférentiel, aux candidats d'origine non-belge engagés dans le parti qui correspond le mieux à ses aspirations personnelles. On peut donc penser que la situation anversoise se rapproche des phénomènes observés dans d'autres pays multiculturels : sans être prépondérante, la variable « ethnicité des candidats » peut néanmoins expliquer une partie des variances observées au niveau de la participation politique, à la fois de la majorité et des minorités ethniques (Tillie, 1994 ; Helly, $1997: 179-185$ ).

La troisième constatation concerne le profil des candidates et des candidats. Les observations relevées au départ de notre étude permettent d'avancer quelques généralisations partielles, mais qui nécessiteront d'être contrastées et enrichies à partir des données relatives à l'ensemble de la Flandre, voire à l'ensemble de la Belgique, dans le cadre de recherches ultérieures. Dans le contexte anversois, on peut tout d'abord souligner que les candidats d'origine étrangère appartiennent essentiellement à une catégorie d'élite ethnique cooptée ${ }^{17}$ par la classe politique locale. Ceci n'est d'ailleurs pas étonnant dans la mesure où, au niveau local, il s'agit d'une méthode utilisée par l'ensemble des partis politiques, et ce vis-à-vis de la plupart des catégories de la population. Par ailleurs, on peut également observer que les candidats aux élections d'origine ethnique sont davantage représentés dans les partis politiques marginaux, au sein des listes dissidentes et des partis politiques contestataires ${ }^{18}$. Il faut également souligner un engagement politique proportionnellement très élevé des femmes. Enfin, on pourra remarquer, pour des raisons en partie liée à l'histoire

17 Sur la notion d'élite ethnique, on pourra se reporter à la définition qu'en propose M.Martiniello (1992 : 93-98).

18 La présence de partis politiques contestataires est une constante dans la politique anversoise. Les minorités ethniques n'échappent pas à ces mouvements. A titre d'exemple, on peut mentionner lors des dernières élections communales, la présence de la liste Boris formée par un collectif $d$ 'artistes de théâtre et au sein de laquelle on pouvait trouver plusieurs Belges d'origine marocaine. La plus médiatisée de ces listes était incontestablement la liste R.O.S.S.E.M, du nom d'un ex-gourou de la finance déchu, et au sein de laquelle on trouvait également plusieurs personnes d'origines ethniques multiples.

REMI 1998 (14) 2 pp. 151-172 
migratoire de la ville, la forte représentation des personnes d'origine marocaine parmi ces candidats ${ }^{19}$.

\section{La structure des opportunités .... et des contraintes politiques}

Pour rendre compte de l'évolution des stratégies participatives des minorités ethniques immigrées, les observateurs académiques ont de plus en plus recours à la notion de structure des opportunités politiques (Statham, 1997 ; Ireland, 1994 ; Wayland, 1993 ; Poinsot, 1994). La structure des opportunités politiques est un concept dérivé de la théorie des nouveaux mouvements sociaux, qui permet précisément d'expliquer l'émergence et l'évolution socio-politique de ces mouvements. Bien que la transposition du concept dans le champ de l'immigration et des relations ethniques reste sujette à caution (Tarrow, 1996), il n'en reste pas moins un concept attrayant, capable de mettre en parallèle l'évolution de la stratégie des groupes sociaux et les modifications de l'espace institutionnel auquel ils sont confrontés.

Dans cette perspective on peut avancer que l'émergence de personnalités d'origine non-belge sur la scène politique formelle a été favorisée par les ouvertures successives qui ont été opérées au niveau du droit de la nationalité belge. Dans la mesure où la citoyenneté politique belge reste une citoyenneté essentiellement liée à l'appartenance nationale, celle-ci ne peut dans le cas des minorités ethniques de nationalité étrangère s'exercer sans passer préalablement par le Rubicon de la naturalisation. Depuis la codification du Droit de la nationalité belge en 1984 et depuis la réforme que cette législation a subi en 1991, la procédure de naturalisation, et donc la possibilité d'accéder à une citoyenneté politique formelle complète a été assouplie et facilitée pour la plupart des non-Belges ${ }^{20}$.

L'existence de telles opportunités ne peut cependant masquer le fait que nombreux sont les étrangers, qui pour de multiples raisons n'obtiennent pas ou ne recourent pas à la naturalisation. Ceci a pour conséquence que la grande majorité de la population de nationalité étrangère reste maintenue en dehors de toute participation politique électorale. Le principe régissant l'accès aux droits politiques, et

19 Avec une population de 21.564 personnes, les Marocains forment la première communauté étrangère d'origine immigrée à Anvers.

20 La nationalité belge peut être obtenue en fonction de quatre procédures d'attribution et de cinq procédures d'acquisition. Soulignons ici que le Code de la nationalité belge de 1991 prévoit une extension du principe du droit du sol comprenant notamment une procédure d'attribution automatique de la nationalité aux enfants de la troisième génération. Les enfants de la deuxième génération peuvent quant à eux bénéficier d'une procédure d'attribution sur demande des parents avant l'âge de douze ans ou utiliser la procédure d'acquisition ouverte aux jeunes de la deuxième génération âgé de plus de dix-huit ans et de moins de trente ans. Le mariage avec un(e) conjoint(e) belge ouvre également la voie à une procédure d'acquisition de la nationalité. Enfin, il existe à côté de ces procédures spécifiques, la procédure classique de naturalisation, qui doit faire l'objet d'une demande solennelle adressée au Parlement, lequel peut au bout du compte octroyer ou refuser la nationalité belge au demandeur. 
principalement au vote et à l'éligibilité, reste fondamentalement attaché au critère de la nationalité belge. Suite à l'adoption du Traité de Maastricht, la situation est cependant en passe d'évoluer sur ce point. L'introduction d'une citoyenneté européenne devrait ouvrir prochainement aux ressortissants de l'Union Européenne résidant en Belgique le droit de participer aux élections locales, en plus du droit de participer aux élections européennes qui a été introduit en Belgique en 1995. La transposition de cette disposition dans le droit belge a fait l'objet de débats intenses dans le monde politique et dans la société civile. L'introduction de la citoyenneté européenne en Belgique a en effet fait naître la crainte d'une remise en cause des équilibres politiques entre les communautés flamande et francophone. Dans l'hypothèse où les nouveaux électeurs apporteraient leur suffrage aux partis politiques appartenant à l'une ou l'autre communauté linguistique, l'équilibre institutionnel dans les communes bilingues risquerait de s'en trouver menacé. Après que le Parlement se soit trouvé dans l'incapacité de trouver la majorité des deux-tiers requise pour réformer la Constitution sur ce point, le gouvernement belge s'est emparé de la question pour tenter d'imposer un compromis - à défaut d'avoir pu trouver un large consensus démocratique - visant à appliquer strictement les dispositions du Traité de Maastricht et de la directive (94/80/CE) en faveur des Européens à l'occasion des élections communales d'octobre 2000. Le second volet du compromis proposé par le gouvernement consistait à promettre une extension du droit de vote et d'éligibilité locale aux étrangers noneuropéens pour la prochaine échéance des élections communales en 2006.

La non-jouissance par les non-Belges des droits de vote et d'éligibilité a, dans le passé, constitué le plus grand frein à une plus grande représentation numérique en leur faveur au sein des assemblées élues. Bien qu'elle soit la plus évidente, la privation des droits politiques fondamentaux ne constitue pas la seule contrainte limitant l'émergence de ces groupes dans les institutions politiques formelles. Pour rendre compte de l'émergence problématique des candidats et des élus issus des minorités ethniques dans les dernières années, il est indispensable de re-situer le contexte politique et électoral plus large qui caractérise la période 1988-1998 en Flandre.

Nous avons souligné plus haut que le rapport de force électoral entre les partis traditionnels d'une part et l'extrême-droite d'autre part subit une évolution constante sur toute la période. Alors que les écologistes et l'extrême-droite progressent significativement, les socialistes, les sociaux-chrétiens et les nationalistes démocratiques sont en net recul. La décennie est donc caractérisée par une mutation profonde du paysage électoral. Par ailleurs, les partis traditionnels s'appuient sur un électorat relativement âgé et déclinant (Swyngedouw, 1992) l'électorat jeune répondant davantage à l'offre politique formulée soit par l'extrême-droite soit par les écologistes, soit encore par des pratiques d'abstention ou de vote blanc. Ces dynamiques à l'oeuvre entre les partis et à l'intérieur des partis a des effets sur la représentation des minorités ethniques. A ce niveau, la politisation extrême du thème de l'immigration imposée par l'extrême-droite a dans un premier temps constitué un facteur d'affaiblissement des candidats issus de ces groupes de population. En effet, certaines tendances au sein des partis traditionnels ont tenté d'imposer l'idée selon laquelle une coloration des listes électorales aurait pour conséquence d'accroître les fuites électorales en faveur de l'extrême-droite (Van de Putte, 1997). Ce phénomène a joué de manière variable au 
sein des différents partis, mais a surtout affecté les partis établis. Alors que les libéraux de droite n'ont présenté de candidat d'origine étrangère sur leurs listes qu'à une seule reprise, lors des élections provinciales de 1991, les autres partis traditionnels (sociauxchrétiens, socialistes, nationalistes démocratiques) n'ont procédé que dans une très faible mesure à de telles inscriptions. Seuls les écologistes ont présenté de tels candidats de manière quasi systématique, sans toutefois que ces derniers soient en toutes circonstances assurés d'une position éligible ${ }^{21}$.

L'inscription des candidats issus des minorités ethniques sur la scène politicoélectorale belge et flamande s'opère dans le cadre d'un système multi-parti relativement fragmenté. Lors du dernier scrutin communal à Anvers, ce sont dix-huit formations qui se sont présentées au suffrage de la population. Cette caractéristique du système belge de compétition entre partis, associée à un système de représentation proportionnelle, crée une configuration du débat politique très particulière, bien éloignée de celle qui prévaut pour les minorités ethniques dans un pays comme la Grande-Bretagne. En Grande-Bretagne, le système bipolaire et la représentation issue du scrutin majoritaire à un tour donnent à ces catégories la possibilité de faire décisivement pencher la balance dans un sens ou dans un autre, et ce particulièrement dans les circonscriptions électorales où existent des phénomènes de concentration résidentielle (Studlar, 1984). Quand bien même les minorités ethniques seraient librement appelées à participer aux élections indépendamment de leur nationalité, la configuration particulière du paysage politique ne leur permettrait probablement pas d'accroître décisivement leur pouvoir électoral.

Il est également important de s'attacher à l'étude des relais dont disposent les partis politiques au sein de tels groupes. La faible proportion de candidats doit en effet être mise en rapport avec la très faible représentation des populations d'origine immigrée parmi les adhérents des partis dominants (Van de Putte, 1997). Les socialistes, les sociaux-chrétiens, les libéraux et les nationalistes démocratiques ne disposent d'aucune assise populaire dans ces milieux ${ }^{22}$. Seuls les écologistes d'Agalev parviennent à structurer leur relais au sein des communautés ethniques en développant quelques éléments d'une politique d'action affirmative visant surtout à courtiser les cadres appartenant à l'élite ethnique. Dans ce contexte, les plus engagés se trouvent dans l'incapacité d'établir des stratégies collectives (sur base ethnique ou non) à l'intérieur de leurs partis respectifs, à la manière de ce que Solomos et Back (1997) observent au sein du parti travailliste britannique. Cette absence de supports internes

21 Voir plus haut le cas de F.Bali.

22 Il n'existe pas de données précises et fiables sur le membership d'origine ethnique des partis politiques. Celui-ci est à l'évidence relativement faible, au point que certains partis tentent de modifier cet état de fait en mettant en oeuvre des stratégies de recomposition de leur base en direction notamment de cette population. Le mouvement Rosa au sein du parti socialiste et le mouvement ID 21 des nationalistes démocratiques de la VU en sont des exemples. A l'heure actuelle, quelques personnes issues des minorités ethniques se sont engagées dans ces dynamiques. 
limite leur capacité de négociation par rapport à la définition des tactiques électorales partisanes, dont ils sont précisément l'un des enjeux.

Nos observations doivent être complétées par une autre remarque. En Belgique, les instances dirigeantes des partis, que ce soit au niveau local ou national, disposent d'un large pouvoir dans la fixation du classement des candidats sur les listes électorales. Dès lors, l'un des terrains de bataille politique essentiel se situe en amont de l'élection proprement dite et consiste à se positionner en place utile à l'intérieur de son propre parti. A Anvers, les candidats d'origine ethnique appartenant aux grandes formations démocratiques ont fait les frais de ce type d'opération à plus d'une reprise. Comme le souligne $\mathrm{Z}$. Gifford : «the most important election is selection » (Z. Gifford in S. Saggar, $1998: x$-xii).

\section{L'impact de la participation et de la représentation des minorités ethniques sur les institutions politiques démocratiques locales.}

Ainsi que nous l'avons souligné, il convient de s'interroger sur la différenciation éventuelle qui peut exister entre un impact politique électoral et un impact qualitatif de fond sur le cours des politiques publiques. Originellement, la distinction entre représentation numérique et représentation substantive a servi à mettre en évidence le fait qu'un accroissement du nombre de candidats et d'élus d'origine ethnique n'était pas nécessairement synonyme d'une plus grande capacité pour les communautés ethniques à imposer une prise en compte de leurs intérêts politiques. Les recherches menées au Canada (Abu Laban, 1997 ; Black and Lakhani, 1997) en France (Geisser, 1997) ou en Grande-Bretagne, (Saggar, 1993 ; 1996) tendent en effet à suggérer que la représentation des personnes issues des minorités ethniques aux sein des institutions politiques élues n'a pas été un facteur décisif d'altération des relations de pouvoir entre les différentes catégories de population.

Dans le cas belge et anversois, on pourrait montrer qu'effectivement le rôle des élus en question a été marginal dans des dossiers cruciaux. Le débat sur le droit de vote des étrangers est peut-être le plus révélateur d'une incapacité à imposer des évolutions politiques décisives au sein de leurs partis. Cependant, il convient d'apporter quelques nuances à cette analyse. On ne peut en effet manquer de constater que les relations entre représentation numérique et représentation substantive sont d'une grande complexité, et dans une certaine mesure autonome. En effet, la prise en compte des intérêts politiques des minorités ethniques peut être faible alors que leur représentation est importante. Comme le montrent Black et Lakhani à travers l'exemple canadien, un certain nombre de candidats d'origine ethnique appartiennent à des partis ouvertement opposés à toute prise en compte spécifique des intérêts des minorités ethniques. Mais, l'exemple inverse est également possible. La représentation des intérêts politiques de ces groupes dans le débat public peut s'accroître en l'absence d'une forte représentation formelle ${ }^{23}$.

23 Catherine Withol de Wenden a pu noter cette éventualité dans le contexte électoral français des années 80 . Bien qu'ils soient privés de droits politiques, elle note que les immigrés jouent 
La situation qui s'impose à Anvers après les élections communales de 1994 s'apparente à ce deuxième cas de figure. Par un paradoxe de la morale politique, les partis politiques au pouvoir au sein de la ville d'Anvers ont tout intérêt pour leur survie électorale à se distancier des thèmes nationalistes et racistes portés par l'extrêmedroite. Après 1994, les partis politiques traditionnels semblent en effet avoir assimilé l'idée qu'un alignement de leur discours sur celui du Vlaams Blok n'est pas porteur en termes de stratégies électorales, et tend à conforter ce parti encore davantage. Par ailleurs, un sondage récent montre que le parti écologiste, qui est perçu comme étant le plus en faveur des droits et des intérêts des minorités, est le seul à être crédité de nouveaux gains électoraux ${ }^{24}$ (Gazet van Antwerpen, 26 mai 1998).

Nous avons souligné dans la première partie que l'extrême-droite est parvenue à Anvers à capter les suffrages de près d'un électeur sur trois lors des dernières élections communales. Dès lors, s'il y a lieu de parler d'une différenciation entre représentation numérique et représentation substantive, cela devrait avant tout concerner les électeurs du Vlaams Blok. Or, paradoxalement, malgré une représentation exceptionnellement élevée de l'extrême-droite, la gestion actuelle de la ville d'Anvers ne semble pas se faire au détriment des populations d'origine immigrée. L'accord politique, scellant la coalition actuelle, est relativement positif en matière de politique du multiculturalisme, et semble même être jugé très positivement par les leaders ethniques (Alsulaiman, 1997).

Bien que les élues politiques d'origine ethnique anversoise n'aient pas toujours été des acteurs majeurs dans les décisions de mener des politiques multiculturalistes, qui figuraient cependant au sein d'un agenda accepté par leurs partis, elles ont malgré tout eu une action décisive sur certains dossiers. On peut à cet effet mentionner les politiques dérivées des propositions introduites par Nahima Lanjri en ce qui concerne la mise en place d'une politique anversoise d'accueil des nouveaux arrivants (réfugiés, candidats réfugiés, personnes arrivées dans le cadre d'un regroupement familial), ou encore en matière de politique d'action affirmative dans la sélection du personnel contractuel de la ville. Dans le même temps, il faut souligner que la portée de cette action est conditionnée par des forces politiques externes, qui en l'occurrence tendent à montrer que malgré une sous-représentation numérique des minorités ethniques, cette population dispose d'une certaine représentation substantive. Les candidates anversoises mettent en évidence, et au delà des divergences qui caractérisent leurs trajectoires politiques personnelles, qu'elles n'ont joué ni un rôle symbolique ni un rôle marginal, mais plus exactement celui d'un adjuvant aux

ce qu'elle appelle un rôle d'acteur politique du second degré : «Ainsi, les immigrés constituent en quelque sorte, des groupes de pression sans le vouloir, parfois sans le savoir (...) et le plus souvent, malgré eux, une force politique par procuration, par partis ou leaders politiques interposés $\gg$ (Withol de Wenden, $1988: 319-320$ ).

24 Il faut toutefois souligner que les sondages pré-électoraux sont sujets à un problème de fiabilité en ce qui concerne l'extrême-droite, laquelle est régulièrement et légèrement désavantagée. Il est en effet relativement plus difficile d'obtenir la collaboration de cet électorat dans le cadre de ce type d'enquête. Par opposition, les partis écologistes sont souvent légèrement avantagés. 
ouvertures multiculturelles de la nouvelle politique anversoise. En d'autres termes, alors que la grande majorité des candidats et des élus issus des minorités ethniques interrogés situent leur action politique dans le cadre d'une lutte en faveur de politiques multiculturalistes, leur capacité d'agir en ce domaine est enserrée dans un espace politique bien déterminé, étroitement dépendant de la dynamique politico-électorale imposée par l'extrême-droite.

Les observations avancées dans cet article apportent des éléments de réponse à la question posée dans la première partie à savoir : la montée de l'extrême-droite à Anvers peut-elle être tenue comme un facteur ayant déclenché une mobilisation réactive au sein des populations d'origine immigrée et a-elle conditionné des stratégies particulières de mobilisation de l'ethnicité ? L'expérience des candidats et des élus d'origine ethnique à Anvers suggère que les deux dynamiques politiques ne sont corrélées que de manière relative. Au niveau de la mobilisation organisationnelle de la société civile, la montée de l'extrême-droite a constitué un facteur incitatif à une mobilisation de résistance, qui a fortement polarisé les termes du débat sur l'immigration. Cependant, au niveau de la scène politique et électorale, l'action des candidats et des élus d'origine ethnique ne se situe pas décisivement par rapport aux termes du débat imposé par l'extrême-droite, malgré l'impact secondaire qui s'impose à eux à travers les logiques actives au sein des partis traditionnels. L'élément mobilisateur de cette élite ethnique politique n'est pas une vision commune de ce que doit être la lutte contre l'extrême-droite ou une divergence qui se cristalliserait sur ce même objet. Il s'agit davantage de convergences et de divergences sur la manière d'organiser l'avancement et le progrès politique et social des communautés ethniques. Il y a là plus qu'une nuance, qui démontre qu'en matière de lutte contre l'extrêmedroite, les acteurs politiques ethniques récusent souvent le jeu de la polarisation.

\section{CONCLUSION}

Dans le contexte d'hyper-politisation de l'immigration, les collectivités issues de l'immigration ne sont en réalité jamais restées complètement passives. En dehors de leurs activités tournées vers les pays d'origine, leur affirmation s'est d'abord située dans le cadre d'une action collective associative au sein de la société civile. Leur affirmation dans l'espace public local et régional ne prendra que plus tardivement la forme d'une action orientée vers la scène politique électorale. Cette affirmation sur la scène électorale se heurte à de nombreux obstacles, dont le plus évident reste la privation du droit de vote et d'éligibilité local pour les personnes de nationalité étrangère. Les prochaines élections communales en Belgique devraient toutefois être l'occasion d'une évolution sinon décisive du moins significative sur le plan symbolique. L'extension de l'électorat, consécutive à la mise en oeuvre des dispositions du Traité de Maastricht relatives à la citoyenneté de l'Union Européenne, amènera une extension de l'électorat anversois se situant aux alentours de 4 à $5 \%$. La question qui est aujourd'hui posée consiste à voir dans quelle mesure cette extension prévisible du corps électoral est à même de peser sur les rapports de force au sein de l'espace politique local et de modifier l'état de la participation et de la représentation des minorités ethniques immigrées.

REMI 1998 (14) 2 pp. 151-172 
La représentation de ces groupes de population est également affectée par la logique de fonctionnement du système de compétition entre les partis, et par la logique interne aux différents partis. Nous avons mis l'accent sur le fait que le poids politique des candidats et des élus d'origine ethnique au sein de leur parti politique respectif n'est pas très important. En tout état de cause, il n'est pas suffisant pour inverser la tendance sur des questions aussi cruciales que la question du droit de vote des nonEuropéens par exemple. Les faibles marges de manoeuvre dont disposent ces candidats dans les grands partis, les craintes liées à la volatilité de certains segments de leur électorat, et les marges de manoeuvre quasi discrétionnaires dont disposent les partis dans la sélection du personnel politique constituent autant de contraintes majeures qui limitent l'affirmation politique des minorités ethniques sur la scène électorale.

Alors que dans un pays comme la Grande-Bretagne, les relations entre les minorités ethniques et la participation politique à travers les organisations partisanes constituent selon S. Saggar (1993) l'un des chapitres les plus documentés de la recherche, la question ne s'est posée en Belgique que très tardivement, et sa prise en compte au niveau des productions académiques en est encore à ses débuts. En tout état de cause, de nombreuses zones d'ombres devront être clarifiées par de futurs travaux, que ce soit au niveau du vote ethnique, de l'impact du système de compétition entre partis, ou encore de la représentation substantive. Nous avons ici envisagé un certain nombre d'hypothèses par rapport à la ville d'Anvers qui nous ont permis de mettre en évidence et de retracer le contexte dans lequel s'opère les débuts d'une affirmation politique des minorités sur la scène électorale. Nous avons également souligné que la stratégie du « cordon sanitaire » mise en place par les partis politiques démocratiques contre l'extrême-droite a contraint ceux-ci à jeter les bases d'une nouvelle politique anversoise en matière d'immigration et de multiculturalisme. L'apparition d'élus politiques locaux appartenant aux minorités ethniques et leur action politique dans le nouveau conseil communal doit donc être mis en rapport avec ce qu'il convient d'appeler un agenda politique libéral de la majorité politique. Au delà de l'inclusion prévue de nouveaux électeurs à l'occasion des prochains scrutins communaux, l'un des grands enjeux consistera à savoir si cette majorité politique dispose des moyens pour le rester.

\section{Références bibliographiques}

ABU LABAN (Y.), « Ethnic Politics in a Globalizing Metropolis : the Case of Vancouver », in THOMAS (T.) éd., The Politics of the City : A Canadian Perspective, Nelson, Scarborough, 1997.

ALSULAIMAN (A.), « De bestuurlijke randvoorwaarden voor de toepassing van het integratieconcept » pp. 183-201, in FOBLETS (M.-C.), et HUBEAU (B.), éds., Nieuwe burgers in de samenleving? Burgerschap en inburgering in België en Nederland, Leuven, Acco, 1997.

BLOMMAERT (J.), et MARTINIELLO (M.), « Ethnic mobilization, Multiculturalism and the political process in two Belgian cities : Antwerp and Liège », Innovation, vol. 9, $\mathrm{n}^{\circ} 1,1996$, pp. 51-73.

CENTRE POUR L'EGALITE DES CHANCES, Rapport Annuel. Annexe Informative, Bruxelles, 1996. 
HELLY (D.), Revue des études ethniques au Québec 1977-1996, Rapport préparé pour Politique, planification et recherches stratégiques et le projet Métropolis, Novembre 1997.

IRELAND (P.), The Policy Challenge of Ethnic Diversity, Harvard University Press, Cambridge Ma, 1994.

MARTINIELLO (M.), Elites, leadership et pouvoir dans les communautés d'origine immigrée, Paris, L'Harmattan, 1992.

MARTINIELLO (M.), «Quelle participation politique » in La Belgique et ses immigrés. Les politiques manquées, ouvrage collectif, De Boeck-Université, Bruxelles, 1997, pp. 103-120.

MARTINIELLO (M.), et SWYNGEDOUW (M.), Où va la Belgique? Les soubresauts d'une petite démocratie européenne, Paris, L'Harmattan, 1998.

POINSOT (M.), L'intégration politique des jeunes issus de l'immigration en France : du débat d'idées aux actions collectives dans la région lilloise, Thèse de doctorat, Institut d'Etudes Politiques, Paris, 1994.

SAGGAR (S.), «Black participation and the transformation of the "race issue » in British Politics ", New Community, vol. 20 (1), 1993, pp. 27-41.

SAGGAR (S.), Race and British Electoral Politics, London, UCL Press, 1998.

STASIULIS (D.K), « Participation by Immigrants, Ethnocultural Minorities in the Canadian Political Process. Discussion Paper », paper presented at the Research Domain Seminar on Immigrants and Civic Participation: Contemporary Policy and Research Issues, Montréal, November 1997.

STUDLAR (D.-T.), The Ethnic Vote, 1983 : Problems of Analysis and Interpretation, New Community, vol. 11, $\mathrm{n}^{\circ} 1-2,1983$, pp. 92-100.

SWYNGEDOUW (M.), «L'essor du Vlaams Blok et d'Agalev ", Courrier Hebdomadaire du CRISP, Bruxelles, 1992, $\mathrm{n}^{\circ} 1362$, p. 42.

SWYNGEDOUW (M.), « Belgium : Explaining the Vlaams Blok - City of Antwerp Relationship ", in HAINSWORTH (P.) Ed., The Extreme Right in Europe and the USA, Pinter, London, 1998.

TAYLOR (R.), « Political science encounters ethnicity », Ethnic and Racial Studies, vol. 19 (4), 1996, pp. 884-895.

TILLIE (J.), Kleurrijk kiezen, NCB, Utrecht, 1994.

VAN DEN BRANDE (A), "Elements for a sociological analysis of the impact of the main conflicts on Belgian political life », Res Publica, vol. IX (3), 1967, pp 459.

WITHOL DE WENDEN (C.), « Les immigrés et la politique », Presses de la FNSP, Paris, 1988.

WITHOL DE WENDEN (C.), « Generational change and political participation in French suburbs », New Community, vol. 21 (1), 1995, pp. 69-78. 


\section{Le paradoxe anversois.}

Entre racisme politique et ouvertures multiculturelles.

\section{Hassan BOUSETTA}

L'article analyse la participation et la représentation politiques des minorités ethniques aux institutions politiques formelles dans le contexte belge, et en particulier dans la ville d'Anvers. La contribution discute les questions méthodologiques et conceptuelles liées à cette problématique et tente d'esquisser un état des lieux aussi complet que possible de cette représentation politique au plan local. Le phénomène de l'émergence politique des candidats et des élus d'origine ethnique est ensuite étudié. L'hypothèse selon laquelle la forte représentation de l'extrême-droite à Anvers a pu en tant que telle constituer un facteur de réaction politique au sein des groupes ethniques d'origine immigrée est examinée. Une dernière problématique soulevée dans cet article a trait à l'impact d'une telle représentation sur le cours des politiques publiques locales à Anvers.

\section{The Antwerp Paradox. Between Political Racism. and Multicultural Perspectives.}

\section{Hassan BOUSETTA}

The article examines the political participation and representation of ethnic minorities within Belgian elected assemblies in the city of Antwerp. The contribution endeavours at providing a comprehensive empirical overview of the issue at stake and discusses a number of methodological and conceptual issues related to the study of the political representation of ethnic minorities. The political emergence of ethnic minority candidates and elected politicians is then studied. One aim of the article is to assess whether the presence of a strongly represented extreme-right, as it is the case in Antwerp, has constituted a factor of political reaction within immigrant ethnic communities in Antwerp. Another issue raised in this contribution is about the impact of the political representation of ethnic minorities on the course of local public policies.

\section{La parádoja de Amberes. Entre racismo político y aperturas multiculturales.}

\section{Hassan BOUSETTA}

Esta contribución tiene por objeto el análisis de la participación y de la representación políticas de las minorias étnicas en las instituciones políticas formales en el contexto belga. Aunque esta representación de las minorías étnicas es todavia un fenomeno cuantitativamente limitado en el seno de múltiples asambleas elegidas por el Estado belga, sin embargo, se trata de un fenómeno político emergente en el contexto de los años noventa. El estudio de esta problemática se concentra en el caso particular de la ciudad de Amberes. La contribución, que presenta un carácter introductorio, discute las cuestiones metodológicas y conceptuales unidas a esta problemática e intenta esbozar un estado de la cuestión tan completo como sea posible de esta representación política en el plano local. A continuación, se estudia el fenómeno de la emergencia política de los candidatos y de los elegidos de origen étnico, desde el punto de vista 
de sus diferentes modalidades en el contexto de Amberes. En efecto, una de las hipótesis que nos ha guiado, en relación con este trabajo, ha consistido en estudiar en qué medida la fuerte representación de la extrema-derecha en Amberes ha podido, como tal, constituir un factor de reacción política en el seno de los grupos étnicos de origen inmigrado. Una ultima problematica planteada en este articulo se refiere al impacto de una representacion, como ésta, en el desarrollo de las políticas públicas en Amberes. 